\title{
Epidemiological Distribution of Reported Cryptosporidiosis cases in Houston, Texas, 2013-2016
}

\author{
Najmus Abdullah, Razina Khayat*, Sudipa Biswas, Hafeez Rehman and Kirstin Short \\ Epidemiology, City of Houston Public Health, Houston, TX, USA
}

\section{Objective}

To demonstrate the demographic and clinical distribution of reported Cryptosporidiosis cases in Houston, Texas, from 2013-2016

\section{Introduction}

Cryptosporidiosis is a diarrheal disease caused by microscopic parasite Cryptosporidium. Modes of transmission include eating undercooked food contaminated with the parasite, swallowing something that has come into contact with human or animal feces, or swallowing pool water contaminated with the parasite. The disease is clinically manifested usually with chronic diarrhea and abdominal cramps. It is found to be more prevalent in immunocompromised patients like HIV and AIDS. Cryptosporidiosis usually causes potentially life-threatening disease in people with AIDS.

\section{Methods}

Data were extracted from the Houston Electronic Disease Surveillance System (HEDSS) from January 1, 2013 to December 31, 2016. A total of 170 confirmed cases received during the study period were analyzed and crossed checked against the national Enhanced HIV/AIDS Reporting System (eHARS) database to examine epidemiological distribution. SAS 9.4 was used to analyze demographics, clinical characteristics as well as transmission factors.

\section{Results}

Approximately, $72 \%$ of the cases were males and $28 \%$ were females. The 35-44 year old age group (37\%) had the highest prevalence. African Americans (49\%) and Hispanics (30\%) had the highest number of confirmed Cryptosporidiosis cases.

133 of the 170 cases, $78 \%$ were previously reported to the eHARS national database as HIV/AIDS cases. Among the cases reported to eHARS, $90 \%$ had AIDS. $10 \%$ of the reported cases were found to be deceased in eHARS database. Among the 170 reported cases, $30 \%$ were hospitalized. Clinical presentations were diarrhea (44\%), followed by abdominal cramps (23\%), and nausea and vomiting $(18 \%)$. Most common transmission factors among cryptosporidiosis cases were found to be men who have sex with men (MSM) (34\%), followed by heterosexual contact with HIV/AIDS patients (14\%), and MSM with Intravenous/Injection drug user (IDU) (5\%). Among the reported cases, $70 \%$ were receiving ongoing medical services for their HIV/AIDS status.

\section{Conclusions}

Cryptosporidiosis in patients with HIV/AIDS diagnosis is mostly prevalent in males, African American adults and those between 35-44 years of age, with common clinical presentations of diarrhea and abdominal cramps. The prevalence of cryptosporidiosis is found to be more common in AIDS patients.

Prevention strategies should be focused on raising awareness among immunocompromised patients with HIV and symptoms of cryptosporidiosis so they get evaluated and treated quickly to prevent conversion to AIDS disease.

\section{Keywords}

Cryptosporidiosis; HIV; Disease Surveillance

\section{Acknowledgments}

Thanks to the City of Houston Health Department.

\section{References}

1. Caccio SM, Pozio E. Advances in the epidemiology, diagnosis and treatment of cryptosptidiosis. Expert Review of Anti-infective Therapy. 2006; 4(3): 429-443.

2. Hunter PR, Nichols G. Epidemiology ad clinical features of cryptosporidium infection in immunocompromised patients. Clinical Microbiology Reviews. 2002 Jan; 15(1): 145-154.

3. http://www.cdc.gov/parasites/crypyo/gen_info/infect.html

\section{*Razina Khayat \\ E-mail: razina.khayat@houstontx.gov}

